\title{
Gratitude and Good Outcomes: Rediscovering Positivity and Perspective in an Uncertain Time
}

\author{
Wen T. Shen ${ }^{1} \cdot$ Julie Ann Sosa ${ }^{1}$
}

Published online: 22 June 2020

(c) Société Internationale de Chirurgie 2020

We are living through a period of unprecedented uncertainty and anxiety. While the Department of Surgery at the University of California, San Francisco (UCSF) has not been overwhelmed with COVID-19 patients, the steady stream of troubling news from around the world over the past few months has been stressful and at times overwhelming. The majority of UCSF surgeons have been sidelined during the pandemic; our trauma and acute care surgeons have remained active, but many of us have sat waiting due to the combination of postponed elective operations and the dearth of COVID-19 patients requiring surgical care [1]. We have closely followed the reports coming out of China, Italy, and especially New York City, horrified by the numbers, stories and images. Many of us have felt a bewildering mix of emotions: grief, frustration, guilt and helplessness as we sit idle, knowing that so many of our colleagues elsewhere are struggling and so many patients are dying despite the best efforts of their caregivers. The fact that the pandemic is disproportionately impacting vulnerable populations and laying bare the inequities of our health care system, coupled with recent renewed awareness of systemic societal racism, have only heightened our sense of despair and anger [2].

The collective morale of our surgeons has been understandably diminished. We therefore sought to identify novel methods to promote positivity and uplift in our Department. The result of our efforts was the initiation of a new Grand Rounds format: "Gratitude and Good Outcomes", or "G and G" Rounds for short. The purpose of " $G$ and G" Rounds is twofold: 1) to allow Department

\footnotetext{
Wen T. Shen

wen.shen2@ucsf.edu

Department of Surgery, UCSF, San Francisco, CA, USA
}

members to publicly express gratitude and 2) to highlight and celebrate examples of outstanding teamwork and surgical skill as demonstrated by our surgeons and their teams. Our inaugural " $\mathrm{G}$ and $\mathrm{G}$ " Rounds took place on Wednesday, May 13th, 2020.

Numerous studies have demonstrated the benefits of expressing gratitude as a means for coping with stress and hardship [3]. The first part of UCSF " $G$ and G" Rounds is devoted to expressing thanks. In our inaugural session, we asked 3 UCSF caregivers on the front lines of the current pandemic to share their experiences and reflections. The 3 speakers included a physician from the Emergency Department, an ICU Nurse, and one of our own Trauma and Critical Care surgeons who spent two weeks serving as a volunteer physician in a COVID-19 hospital in New York City. The inclusion of team members from outside of surgery was a primary goal; we recognize that non-surgeon physicians, nurses and other staff have been shouldering the burden of preparing and caring for COVID-19 patients, placing themselves at significant personal risk, and we wanted to express our gratitude for their selflessness and service. We also know that surgery is a team sport that requires the expertise, dedication, and input of multiple other team members, whom we don't acknowledge or celebrate often enough. Each of our speakers shared examples of resilience, creativity and hope in the midst of chaos and upheaval. Our UCSF surgeon who traveled to New York City brought back a message of gratitude from our colleagues there: a heartfelt "Thank You" for simply caring and for sending some of our best nurses and doctors to help.

The second part of our inaugural " $\mathrm{G}$ and $\mathrm{G}$ " Rounds consisted of two non-COVID-19-related cases that posed immense challenges to our surgeons and resulted in excellent outcomes for the patients involved. We 
deliberately selected cases that required a multidisciplinary effort and showcased the phenomenal work of our residents, nurses and colleagues from a wide variety of specialties. One of our cases concluded with a note of thanks from the grateful patient himself, who had spent 8 months in the hospital and endured multiple operations, endoscopic procedures, ICU stays and 2 Code Blue events. Video footage of him healthy and active was a testament to his incredible resilience and the outstanding care that he received.

We view the "Good Outcomes" portion of "G and G" Rounds to be complementary to our traditional Morbidity and Mortality Conference. Surgeons should always recognize and learn from our mistakes, and " $M$ and $M$ " is a fundamental component of our culture and ethos. However, we can also learn from the positive results of our efforts: the examples of great teamwork, surgical skill, ingenuity and overall outstanding care that we continually strive to provide. Surgeons and their teams achieve great results every day, and frankly, we usually take these outcomes for granted. We believe that taking some time to recognize and celebrate good work, especially in the midst of turmoil and hardship, should be an essential component of our training and practice.

Expressing gratitude and highlighting positive outcomes are not new concepts for Departments of Surgery. We ourselves have previously devoted portions of Grand Rounds to "great saves" and outstanding teamwork. Our unprecedented recent events, however, inspired us to create a new Grand Rounds format and to establish a novel moniker that we hope will stick: "Gratitude and Good Outcomes", or "G and G". We anticipate that "G and G" Rounds will become a mainstay of our Grand Rounds rotation and hope that it is adopted by other Departments and institutions. Through this new format, we aim to provide perspective and positivity as we move forward in uncertain times. We are incredibly lucky to be members of this profession, and we should never lose sight of all that we have.

\section{References}

Lancaster EM, Sosa JA, Sammann A, Pierce L, Shen W, Conte MC, Wick EC (2020) Rapid response of an academic surgical department to the COVID-19 pandemic: implications for patients, surgeons, and the community. J Am Coll Surg 230(6):1064-1073

Webb Hooper M, Nápoles AM, Pérez-Stable EJ (2020) COVID-19 and racial/ethnic disparities. JAMA. https://doi.org/10.1001/ jama.2020.8598

Melnyk BM, Kelly SA, Stephens J, Dhakal K, McGovern C, Tucker S, Hoying J, McRae K, Ault S, Spurlock E, Bird SB (2020) Interventions to improve mental health, well-being, physical health, and lifestyle behaviors in physicians and nurses: a systematic review. Am J Health Promot. https://doi.org/10.1177/ 0890117120920451

Publisher's Note Springer Nature remains neutral with regard to jurisdictional claims in published maps and institutional affiliations. 